\title{
Classifying Chimpanzee (Pan troglodytes) Landscapes Across Large-Scale Environmental Gradients in Africa
}

\author{
Kelly L. van Leeuwen ${ }^{1,2,3}$ (D) Ross A. Hill ${ }^{1,2}$. \\ Amanda H. Korstjens ${ }^{1,2,3}$
}
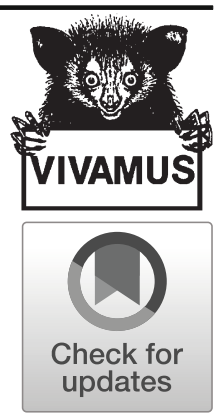

Received: 1 March 2019 / Accepted: 25 May 2020 / Published online: 2 July 2020

(C) The Author(s) 2020

\begin{abstract}
Primates are sometimes categorized in terms of their habitat. Although such categorization can be oversimplistic, there are scientific benefits from the clarity and consistency that habitat categorization can bring. Chimpanzees (Pan troglodytes) inhabit various environments, but researchers often refer to "forest" or "savanna" chimpanzees. Despite the wide use of this forest-savanna distinction, clear definitions of these landscapes for chimpanzees, based on environmental variables at study sites or determined in relation to existing bioclimatic classifications, are lacking. The robustness of the forest-savanna distinction thus remains to be assessed. We review 43 chimpanzee study sites to assess how the landscape classifications of researchers fit with the environmental characteristics of study sites and with three bioclimatic classifications. We use scatterplots and principal components analysis to assess the distribution of chimpanzee field sites along gradients of environmental variables (temperature, rainfall, precipitation seasonality, forest cover, and satellite-derived Hansen tree cover). This revealed an environmental continuum of chimpanzee study sites from savanna to dense forest, with a rarely acknowledged forest mosaic category in between, but with no natural separation into these three classes and inconsistencies with the bioclimatic classifications assessed. The current forest-savanna dichotomy therefore masks a progression of environmental adaptation for chimpanzees, and we propose that recognizing an additional, intermediate "forest mosaic" category is more meaningful than focusing on the ends of this environmental gradient only. Future studies should acknowledge this habitat continuum, place their study sites on the forest-savanna gradient, and include detailed environmental data to support further attempts at quantification.
\end{abstract}

Handling Editor: Erin G. Wessling

Electronic supplementary material The online version of this article (https://doi.org/10.1007/s10764-02000164-5) contains supplementary material, which is available to authorized users.

Kelly L. van Leeuwen

kvanleeuwen@bournemouth.ac.uk

Extended author information available on the last page of the article 
Keywords Ape $\cdot$ Climate $\cdot$ Ecological transition $\cdot$ Hominid $\cdot$ Nomenclature $\cdot$ Vegetation

\section{Introduction}

Nonhuman primates are found across a wide variety of landscapes, but species are sometimes categorized in terms of their preferred or primary natural habitat (Meijaard 2016). Categorizing primates in terms of their preferred habitat largely ignores their flexibility in the landscapes that they use as a consequence of environmental gradients and/or anthropogenic disturbances (Chapman and Peres 2001; Estrada et al. 2012; McKinney 2015; Meijaard 2016). Such flexibility in habitat selection is considered important for primate survival in response to anthropogenic and natural changes to their preferred habitats (Estrada et al. 2017; Galán-Acedo et al. 2019). However, to anticipate how species may respond to the major changes that their landscapes are currently undergoing, we require a good understanding of the landscape-scale habitat requirements and preferences of primates (e.g., Galán-Acedo et al. 2019), as well as clear classifications of the habitat types and landscapes used by various primate species. Although categorization of primate habitat is typically a simplification of the natural world, science benefits from clear and detailed categories in order to provide structure and consistency among researchers.

An apparent solution to classifying primate landscapes would be to use existing bioclimatic classifications of equatorial Africa. However, to date no universally accepted climate and vegetation classification scheme exists, as scientists typically classify habitats according to one or more key climate and vegetation characteristics (developing vegetation formations, ecoregions, or biomes) most relevant to their study (TorelloRaventos et al. 2013). Each environmental classification approach (e.g., WWF ecoregions: WWF 2018; the Koppen-Geiger system: Peel et al. 2007; bioclimatic types: Blasco et al. 2000; White's Vegetation Map of Africa: White 1983) has advantages and disadvantages, and the different approaches often result in different landscape categorizations. These inconsistencies make it difficult to decide which climate or vegetation framework to use to classify primate habitats.

Classifying landscapes is complicated because they are spatially complex and heterogeneous with various different types of vegetation (e.g., forest, woodland, grassland) and differing degrees of anthropogenic disturbance (Arroyo-Rodríguez and Fahrig 2014; McGarigal 2002; McGarigal et al. 2009). Complexities furthermore exist within vegetation types. For example, the term "forest" is used for various types of forest vegetation such as rainforests, dry forests, montane forest, evergreen forest, mixed forest, and secondary forest, depending on local habitat conditions (Bryson-Morrison et al. 2016; Collins and McGrew 1988; Oliveras and Malhi 2016; White 1983). Similar variations are observed for other vegetation types, such as woodland, swamp, and savanna grassland (Collins and McGrew 1988; Hernandez-Aguilar 2009; White 1983). Landscapes differ not only in their vegetation cover (i.e., the presence and relative abundance of different vegetation types), but also in their vegetation spatial arrangement (i.e., the spatial layout of vegetation types), in their climate (e.g., rainfall, temperature, length of the dry season), and consequently in their resource quality, abundance, and distribution (Arroyo-Rodríguez and Fahrig 2014; Hunt and McGrew 2002). Quantitative data on these landscape-scale differences can provide an alternative approach to using existing bioclimatic categorization schemes in classifying primate habitats. 
One primate species that occupies a wide range of habitats is the chimpanzee (Pan troglodytes). Chimpanzees are traditionally characterized as being adapted primarily to inhabit forest environments, and are referred to as "forest chimpanzees" or "forest dwellers" (as reviewed in Hunt and McGrew 2002; Kortlandt 1983; McGrew et al. 1981; Russak 2013). Long-term chimpanzee research has, however, shown that chimpanzees are also well-adapted to inhabit forest mosaics and more open savannawoodland habitats (e.g., Heinicke et al. 2019; Hunt and McGrew 2002; Kortlandt 1983; McGrew et al. 1981; Wessling et al. 2018a, b). Researchers studying chimpanzees in savanna-woodland landscapes classify the chimpanzees they study as "savanna chimpanzees," "open-habitat chimpanzees," or "savanna-dwellers" (e.g., McGrew et al. 1981; Piel et al. 2017; Pruetz et al. 2002).

In the literature on chimpanzees, there is a strong dichotomy between forest and savanna chimpanzees, and researchers often use this distinction to explain the behavioral differences that are observed for chimpanzees in savannas as compared with those in more forested environments. For example, chimpanzees in savannas dig holes for drinking water (Hunt and McGrew 2002), use caves and soak in pools for thermoregulation (Pruetz 2007; Pruetz and Bertolani 2009), consume a wider range of dietary items than chimpanzees in more forested habitats (Hernandez-Aguilar et al. 2007; McGrew et al. 1988), use tools for hunting (Pruetz and Bertolani 2007), move and forage at night (Pruetz 2018), and use energy minimizing behavioral strategies (Pruetz and Bertolani 2009). Chimpanzees in savannas thus display several unique behaviors and these are typically explained as a result of coping with the particular stressors of their environments, which are considered to be much more climatologically and ecologically harsh than forest habitats (Moore 1996; Pruetz and Bertolani 2009; Wessling et al. 2018a). Furthermore, the savanna-woodland landscapes of savanna chimpanzees are hypothesized to closely resemble the environments of early hominins (Moore 1996; Pruetz and Bertolani 2009). Given their close relatedness to humans, the behavioral responses of chimpanzees to savanna environments may provide unique insights into the selective pressures that shaped hominin evolution (Moore 1996; Pruetz and Bertolani 2009).

Despite the wide use of the forest and savanna chimpanzee distinction, the exact environmental conditions under which researchers identify a landscape occupied by chimpanzees as a "forest" or a "savanna" (and thereby attach these labels to the chimpanzees themselves) remain unclear. Quantitative definitions of these chimpanzee landscapes, either based on environmental variables at study sites or determined in relation to existing bioclimatic classifications, are lacking. It therefore remains unknown whether the currently used labels of forest and savanna chimpanzees are robust and supported by empirical evidence. Furthermore, as the forest-savanna transition forms a natural environmental gradient (Thomas 2016), it could be argued that focusing on the two end points (forest and savanna) masks a progression of environmental adaptation of chimpanzees. Therefore, perhaps there is a justification for a focus on more intermediate categories (e.g., forest mosaics) and an acknowledgment of the gradient itself.

Quantitatively categorizing chimpanzee habitats and providing exclusive and nonoverlapping definitions for these classifications could ensure greater consistency and 
clarity for future comparative studies. This could include investigations of the sources and functions of chimpanzee behavioral variability across sites and habitats (Moore 1992), chimpanzee landscape requirements and constraints across environments (Wessling et al. 2019; Wessling et al. unpubl. data), chimpanzee susceptibility and adaptability to future habitat alterations and climate change throughout their range (Pruetz 2018), and the selective pressures influencing human evolution (Copeland 2009; McGrew et al. 1981; Moore 1992; Pruetz and Bertolani 2009).

Chimpanzee researchers typically describe their study landscapes in terms of the environmental aspects that potentially drive chimpanzee behavior and ecology (e.g., Collins and McGrew 1988; Kortlandt 1983; McGrew et al. 1981; Moore 1992). Chimpanzee savanna landscapes are generally considered to have hotter and drier climates, limited forest cover, and less floristic diversity, and to be scarcer and more seasonal in their resources as compared with chimpanzee forest landscapes (Hunt and McGrew 2002; Kortlandt 1983). McGrew et al. (1981) and Moore (1992) were among the first to attempt to classify chimpanzees according to their habitat based on these landscape-scale differences, and argued that vegetation cover, amount and distribution of rainfall, and temperature are the most important factors for chimpanzee landscapebased classifications [ $c f$. Kortlandt (1983), who argued that floristic diversity was also important]. Nonetheless, the resulting comparisons of vegetation composition and climate across chimpanzee sites did not provide exact definitions to quantitatively distinguish savanna from forest landscapes for chimpanzees on the basis of these environmental variables. To our knowledge, no further attempts to develop clear definitions have been published since then. Therefore, a thorough review of literature describing chimpanzee habitat provides an opportunity to develop a consistent landscape-based classification scheme relevant to chimpanzee distribution.

In this study, we review 43 well-documented chimpanzee study sites to establish if the classifications (savanna or forest) given to these sites by researchers are consistently reflected in environmental conditions (climate and vegetation cover) at those sites, which could lead to quantitative definitions. We furthermore compare chimpanzee researcher classifications of study sites with three detailed and commonly used environmental zonations of equatorial Africa: the WWF terrestrial ecoregions (WWF 2018), White's Vegetation Map of Africa (White 1983), and the bioclimatic classification of Whittaker (Ricklefs 2008; Whittaker 1975). Finally, we investigate patterns in the environmental data, and assess the fit of each chimpanzee study site to the prevailing environmental gradients.

\section{Methods}

\section{Study Species}

In the wild, chimpanzees occupy a wide variety of environments ranging from dense forests to savannas, and this variety of habitats is observed across all four chimpanzee subspecies (i.e., the western chimpanzee, Pan troglodytes verus; the Nigeria-Cameroon chimpanzee, $P$. t. ellioti; the central chimpanzee, $P$. t. troglodytes; and the eastern chimpanzee, P. t. schweinfurthii: e.g., Humle et al. 2016). We collected data on the range of habitats described for all four subspecies for analysis. 


\section{Data Collection}

We conducted a systematic search of all literature on chimpanzee field sites in their natural environments available in Web of Science up to December 2017 (i.e., peerreviewed literature: e.g., academic journals, articles, books, and book chapters). In three cases, we obtained additional information from a nongovernmental organization (NGO) report (Howard 1991), a state agency report (Bastin 1996), and a PhD thesis (Russak 2013); we also added some information based on personal communications with researchers [Electronic Supplementary Material (ESM) Appendices S1 and S2]. Specifically, we searched for publications that provided information on the vegetation cover and climate of chimpanzee study sites using the key words "landscape", "habitat", "environment", "vegetation", and "climate" in combination with "chimpanzee", and by specifically searching for the identified chimpanzee study sites by name. We included only sites that encompassed vegetation data to allow for landscape class distinctions, and either climate data or location [so that we could derive climate data from WorldClim climate models (Fick and Hijmans 2017), based on the African weather station network]. Our sample thus provided an exhaustive list of chimpanzee study sites with sufficient environmental information to quantify chimpanzee landscapes. For each chimpanzee study site, we recorded the name, location (GPS-referenced), current environment (i.e., climate and vegetation), landscape class, and the descriptive information provided in the literature [ESM Appendices S1-S3].

With regard to landscape classifications, we categorized field sites as forests or savannas based on the specific use of the terms "forest" or "savanna" by researchers in their labeling either of the chimpanzees themselves, or in most cases, the landscape at their study sites. For sites categorized as forests, we recognized a further distinction between dense forests and forest mosaics based on the explicit use of the terms "forest" or "mosaic" by researchers in their labeling of their field sites. We used the general

Table I Landscape descriptions and key words used by researchers studying chimpanzees to distinguish between forest and savanna sites, and within forest sites to separate dense forest from forest mosaic sites

Landscape Description

1. Savanna Landscapes that are hot, dry and open, dominated by woodland and grassland vegetation types, and with minimal forest cover. Chimpanzees described as "savanna," "savanna-dwelling," or "dry-habitat" chimpanzees.

2. Forest Landscapes that are generally cool, humid, and wet, and characterized by forest vegetation types. Chimpanzees described as "forest chimpanzees" or "forest-dwellers."

2a. Forest Forest landscapes dominated by a mosaic of forest and other vegetation types (e.g.,

mosaic woodland, grassland, cultivated fields). The mosaic character of the site is either explicitly mentioned or described. Chimpanzees sometimes described as "woodland" chimpanzees. Mosaic landscapes are often described as originating from dense forests that have been disturbed, either by anthropogenic influences and/or natural processes and disasters. Landscapes are often referred to as forest-agricultural mosaics, forest-farm mosaics, forest-woodland mosaics, or forest-savanna mosaics, clearly indicating that forest is not the only dominant type of vegetation.

2b. Dense Forest landscapes dominated by forest vegetation types, with minimal other vegetation types forest present (e.g., woodland, savanna grassland, swamp). Chimpanzees often described as "forest" chimpanzees. 
descriptions associated with these categories found in the published literature (Table I) to categorize nine further chimpanzee study sites where authors did not use explicit terminology or where their usage of terminology was inconclusive (i.e., using more than one term in labeling the landscape at their study site). Here, we applied category labels based on descriptions of vegetation types and cover (Table I: $N=4$ "forest mosaic"; $N=5$ "dense forest"). While the terms "forest" and "savanna" are often applied directly to the chimpanzees, the term "mosaic" is only ever applied to the landscape and not used for the chimpanzees themselves in the literature that we assessed. In this study, we applied these categories for indicative purposes only in searching for possible quantitative category boundaries; we did not use these categories for statistical analyses.

With regard to vegetation, we recorded the presence of specific vegetation types (e.g., forest, woodland, bamboo, bushland, swamp, cultivated fields, grassland) and the vegetation cover (i.e., the relative abundance of different vegetation types) as given by the original researchers for each chimpanzee study site. In addition, we used Landsat derived maps of global tree cover (Hansen et al. 2013), imported into R (version 3.5.2, package "raster," function "extract"; Hijmans and Elith 2017), to extract the overall percentage of tree cover within a $5-\mathrm{km}$ radius of the GPS-referenced location of chimpanzee study sites. Hansen et al. (2013) defined trees as all vegetation taller than $5 \mathrm{~m}$ in height. We chose a $5-\mathrm{km}$ buffer to approximate chimpanzee home range size $(N$ $=20$, range $=8-86 \mathrm{~km}^{2}, 5-\mathrm{km}$ buffer $=78.5 \mathrm{~km}^{2} ; 85 \%$ of sites fall within this range: ESM Appendices S1-S3). Using this 5-km buffer is likely to include the tree cover of the complete chimpanzee home range at a site. The closest chimpanzee study sites in our analyses (Bossou and Nimba, Guinea: Koops et al. 2012; Matsuzawa et al. 2011) are ca. $5 \mathrm{~km}$ apart. Values for Hansen tree cover differ from the field-derived values of forest cover, woodland cover, etc., which are vegetation type specific. Hansen tree cover data incorporate any woody vegetation (including forest, woodland, and swamp) and provide an objective measurement of tree cover across a wider range of vegetation types. It could, therefore, be argued that the Hansen tree cover layer provides less informative data for chimpanzee study sites than the vegetation type information reported by the original researchers, as the Hansen tree cover layer was developed for a global analysis of forest cover loss (Hansen et al. 2013), rather than being specifically designed to identify African vegetation types important to chimpanzees.

With regard to climate, we noted the mean annual precipitation $(\mathrm{mm})$, mean annual temperature $\left({ }^{\circ} \mathrm{C}\right)$, total number of dry months per year (i.e., months with $<100 \mathrm{~mm}$ of rainfall: Hunt and McGrew 2002; Matsuzawa et al. 2011; Russak 2013), and length of the longest consecutive dry season (as there is more than one dry season at some sites) for each chimpanzee study site. In cases where the publications we used did not include these climatic data for a specific site, we used WorldClim Global Climate Data (Fick and Hijmans 2017), imported into R, to extract these climatic details with a 1-km buffer around the GPS-referenced study site location (Hijmans et al. 2005).

\section{Data Analyses}

For each chimpanzee study site, we created an overview of the researcherspecified landscape class (i.e., dense forest, forest mosaic, or savanna), vegetation cover, and climate of the site (Table II, ESM Appendices S1-S3). If different 
publications for the same study site described different data, we selected the most site-specific, longest duration, and recent data. We then used scatterplots (IBM SPSS Statistics, version 22) to visually inspect the environmental data from chimpanzee study sites and assess whether the landscape classification of study sites from chimpanzee researchers reflected natural groupings within these environmental variables [i.e., mean annual temperature $\left({ }^{\circ} \mathrm{C}\right)$, mean annual rainfall $(\mathrm{mm})$, length of the longest consecutive dry season (\#, number of months), total number of dry months (\#), forest cover (\%), and Hansen tree cover (\%)]. We used only the amount of forest cover (e.g., as opposed to woodland and grassland cover) to characterize the vegetation cover at sites because of the inherent importance of forested vegetation to chimpanzees (Hunt and McGrew 2002), and because this was the most consistently recorded vegetation cover in chimpanzee literature. Although other vegetation types such as woodland are also considered important for chimpanzees, especially in less forested habitats (e.g., Piel et al. 2017; Pruetz and Bertolani 2009; Pruetz et al. 2008), their coverage across chimpanzee study sites is less consistently reported in the literature so we did not include it in our analyses other than as a part of the Hansen tree cover measure. We also plotted all chimpanzee study sites, labeled with their researcher classifications, against the Whittaker Biome Diagram (Ricklefs 2008; Whittaker 1975), the WWF terrestrial ecoregions (WWF 2018), and White's Vegetation Map of Africa (White 1983; IBM SPSS Statistics or ArcMap, version 10.2.2) to assess the consistency of chimpanzee researcher classifications against bioclimatic categorization schemes. Finally, we used principal components analysis (PCA) to evaluate natural patterns in the environmental data of chimpanzee study sites and assess the distribution of sites across the prevailing environmental gradients. We used a factor analysis based on mean annual temperature $\left({ }^{\circ} \mathrm{C}\right)$, mean annual rainfall $(\mathrm{mm})$, length of the longest consecutive dry season (\# of months), total number of dry months (\#), forest cover (\%), and Hansen tree cover (\%) with varimax rotation (IBM SPSS Statistics). We included only study sites with available data for all vegetation and climate variables in the PCA $(N=32)$. We labeled sites with the classification used by chimpanzee researchers in scatterplots of (regression) component scores, but we did not use these categorizations as input for the PCA.

\section{Ethical Note}

This study did not include any direct research on animal or human subjects. The authors declare that they have no conflicts of interest.

Data Availability All data generated or analyzed during this study are included in this published article and its supplementary information files.

\section{Results}

We identified 43 chimpanzee field study sites across equatorial Africa for which publications provided sufficient vegetation cover and climate data to quantify the 
Table II Landscape classifications of 43 chimpanzee study sites based on terminology or descriptions of vegetation cover by researchers studying chimpanzees (Table I)

1. Savanna sites $(N=9) \quad$ 2a. Forest mosaic sites $(N=12)$

Bafing (Mali)

Comoé (Ivory Coast)

Fongoli (Senegal)

Ishasha River (DRC)

Issa Valley (Tanzania)

Kasakati (Tanzania)

Mount Assirik (Senegal)

Semliki WR (Uganda)

Ugalla (Tanzania)

Bakoun (Guinea)
Bossou (Guinea)
Bulindi (Uganda)
Caiquene-Cadique (Guinea-Bissau)
Gashaka Gumti NP (Nigeria)
Gombe NP (Tanzania)
Kpala (Liberia)
Lac Tumba Landscape (DRC)
Lagoas de Cufada NP (Guinea-Bissau)
Mahale Mountains NP (Tanzania)
Tenkere (Sierra Leone)
Tongo (DRC)

Tongo (DRC) 2b. Dense forest sites $(N=22)$

\author{
Budongo FR (Uganda) \\ Bwindi-Impenetrable NP (Uganda) \\ Dzanga-Ndoki NP (CAR) \\ Gishwati (Rwanda) \\ Goualougo Triangle (Republic of Congo) \\ Ituri FR (DRC) \\ Kahuzi-Biega NP (DRC) \\ Kalinzu FR (Uganda) \\ Kibale NP (Uganda) \\ La Belgique (Cameroon) \\ Loango (Gabon) \\ Lopé NP (Gabon) \\ Minkébé NP (Gabon) \\ Monte Alén NP (Equatorial Guinea) \\ Moukalaba-Doudou (Gabon) \\ Ndoki-Likouala (Congo) \\ Ngel Nyaki FR (Nigeria) \\ Ngotto Forest (CAR) \\ Nimba Mountains (Guinea) \\ Odzala NP (Republic of Congo) \\ Sapo (Liberia) \\ Taï NP (Ivory Coast)
}

NP = National Park; FR = Forest Reserve; and WR = Wildlife Reserve (Inskipp 2005; Russak 2013). References are provided in ESM Appendices S1 and S2.

landscape. These 43 sites represent a broad geographical and environmental range of chimpanzee distribution. Based on terminology or descriptions of vegetation cover used by researchers, we could separate the 43 sites into forests $(N=34)$ and savannas $(N=9$; Table II). We could furthermore separate the forest sites into dense forests $(N=$ 22) and forest mosaics ( $N=12$; Table II).

The 43 chimpanzee study sites differed widely in their vegetation composition, with sites containing one to six different vegetation types of varying proportions and sizes [ESM Appendices S1 and S3]. Reported vegetation and land cover types included forest, swamp, woodland, mangrove, bamboo, bushland, shrubland, terrestrial herbaceous vegetation, savanna grassland, cultivated fields, beach, lava flows, rocky outcrops, and bare land. Although most studies specified the specific vegetation types present at their field site, only a few quantified the amount of each vegetation type, for example by describing the area $\left(\mathrm{km}^{2}\right)$ or relative coverage (as \% of total area). Many

Fig. 1 Relationships of vegetation cover and climate at chimpanzee study sites, labeled with the landscape classifications used by researchers. (a) Mean annual temperature $\left({ }^{\circ} \mathrm{C}\right)$ vs. forest cover (\%, defined by researchers). (b) Mean annual temperature vs. Hansen tree cover (\%, satellite-derived; Hansen et al. 2013). (c) Mean annual rainfall (mm) vs. forest cover. (d) Mean annual rainfall vs. Hansen tree cover. (e) Length of the longest consecutive dry season (\# months) vs. forest cover. (f) Length of the longest consecutive dry season vs. Hansen tree cover. (g) Total number of dry months vs. forest cover. (h) Total number of dry months vs. Hansen tree cover. 

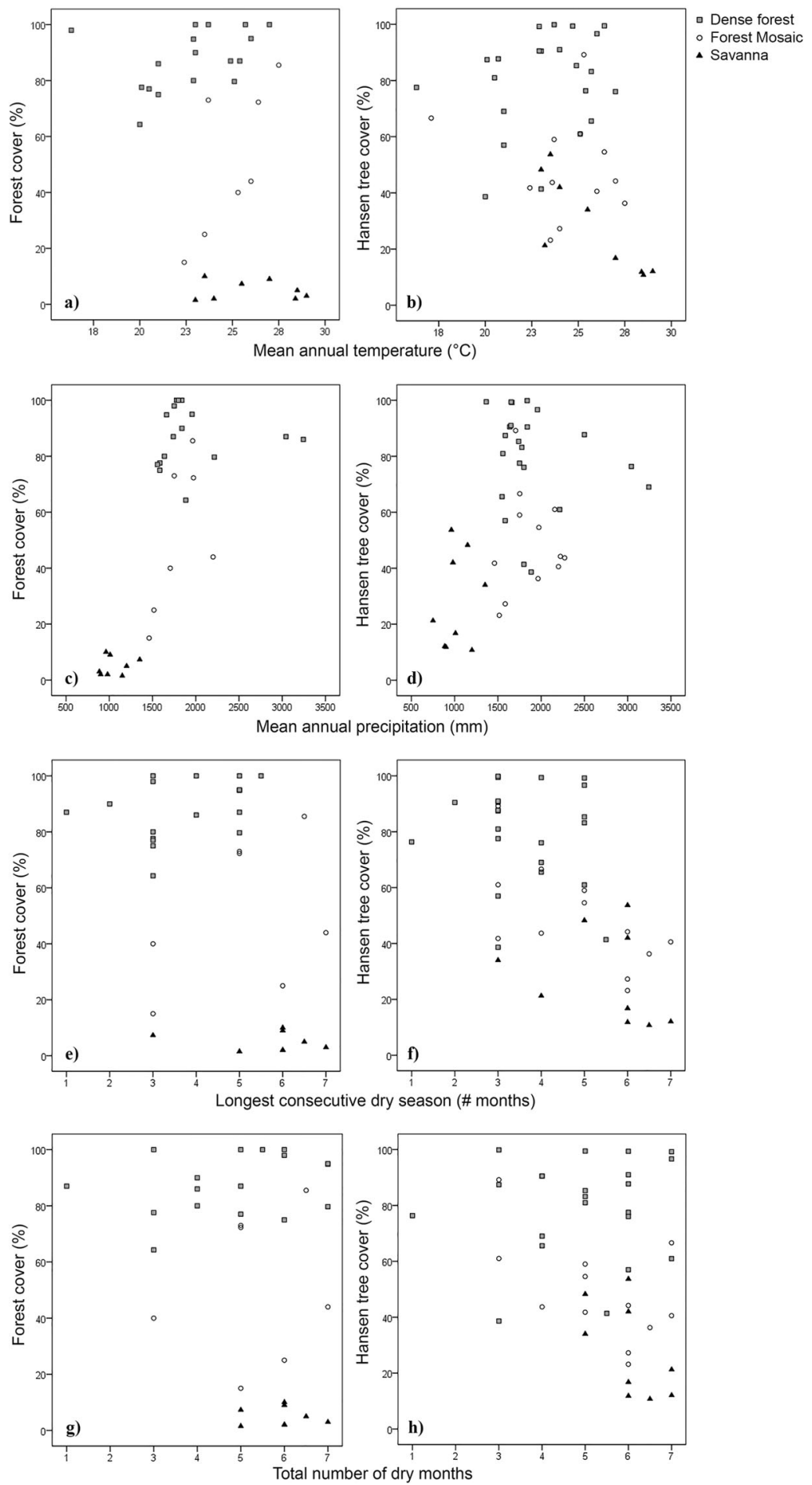
authors only quantified the specific proportion of forest within their study area. Forest was also the only type of vegetation consistently present across all sites. Forest cover ranged 1.5-100\%, and Hansen tree cover ranged 10.7-99.9\%.

The 43 chimpanzee study sites varied considerably in their climatic conditions [ESM Appendices S2 and S3]. Mean annual temperature ranged $16.3-29.0^{\circ} \mathrm{C}$, mean annual precipitation 750-3244 mm, length of longest consecutive dry season 1-7 months, and total number of dry months 1-7 months.

Researcher-specified landscape classes of chimpanzee study sites showed no natural groupings when we plotted and compared all pairs of environmental variables together (Fig. 1, ESM Appendix S4). Within our dataset, researcherclassified savanna sites could be separated from forest sites only in the biplot of annual rainfall $(<1360 \mathrm{~mm} / \mathrm{yr})$ and forest cover $(<12.5 \%$; Fig. 1c). Similarly, within our data set a distinction could be suggested between researcherclassified dense forest and forest mosaic sites based on a relationship between forest cover and either annual temperature (Fig. 1a) or length of the longest consecutive dry season (Fig. 1e). Overlap existed among the chimpanzee landscape categories for all other environmental variables we assessed, and there was no clear separation of dense forest, forest mosaic, and savanna chimpanzee study sites across any of the sets of variables.

Researcher classifications of chimpanzee dense forest, forest mosaic, and savanna sites did not match consistently with the three selected bioclimatic classifications (Fig. 2 and Table III). The WWF terrestrial ecoregions (WWF 2018), White's Vegetation Map of Africa (White 1983), and the Whittaker Biome Diagram (Ricklefs 2008; Whittaker 1975) differed in their landscapes and environmental distinctions, and all three classification schemes placed some of the 43 chimpanzee study sites differently. None of the selected vegetation and climate classification schemes agreed perfectly with the savanna and forest distinction that researchers have used: chimpanzee dense forest, forest mosaic, and savanna sites were placed in various, nonmutually exclusive habitat classes across the maps (Fig. 2 and Table III).

The PCA showed a continuum of chimpanzee study sites along an environmental gradient from savanna to forest (Fig. 3). Factor analysis identified two principal components with an eigenvalue of at least one, with component 1 accounting for $55.7 \%$ and component 2 accounting for $17.7 \%$ of the total variance in the six input environmental variables (Table IV). Component 1 was positively correlated with forest cover, mean annual rainfall, and Hansen tree cover, while component 2 was positively correlated with mean annual temperature, length of the longest consecutive dry season, and total number of dry months (Fig. 3 and Table IV). All researcher-classified savanna sites fell at one end of the environmental continuum (Fig. 3, left panels), and all but one dense forest site fell at the other end of the continuum (Fig. 3, right panels), while forest mosaic sites fell in the middle with some overlap with both dense forest and savanna sites (Fig. 3). Whereas the bottom right panel of Fig. 3 included only researcher-classified dense forest sites, the top right panel included both dense forest and forest mosaic sites, suggesting a degree of overlap between these two classes in forest cover, dry season duration, temperature, rainfall, and Hansen tree cover. 


\section{Discussion}

Based on explicitly used terminology or descriptions of vegetation cover by researchers in the published literature, chimpanzee study sites can be separated into forests and savannas. We furthermore recognized a further distinction within chimpanzee forest sites between dense forest and forest mosaic landscapes based on terminology or environmental field site descriptions. Within our data set chimpanzee researchers typically classified their sites as savannas as opposed to forest when rainfall was $<1360 \mathrm{~mm}$ and forest cover was $<12.5 \%$, and categorized dense forest and forest mosaic sites based on an interaction between forest cover, annual temperature, and dry season duration. Nevertheless, our analyses overall showed no natural groupings in the
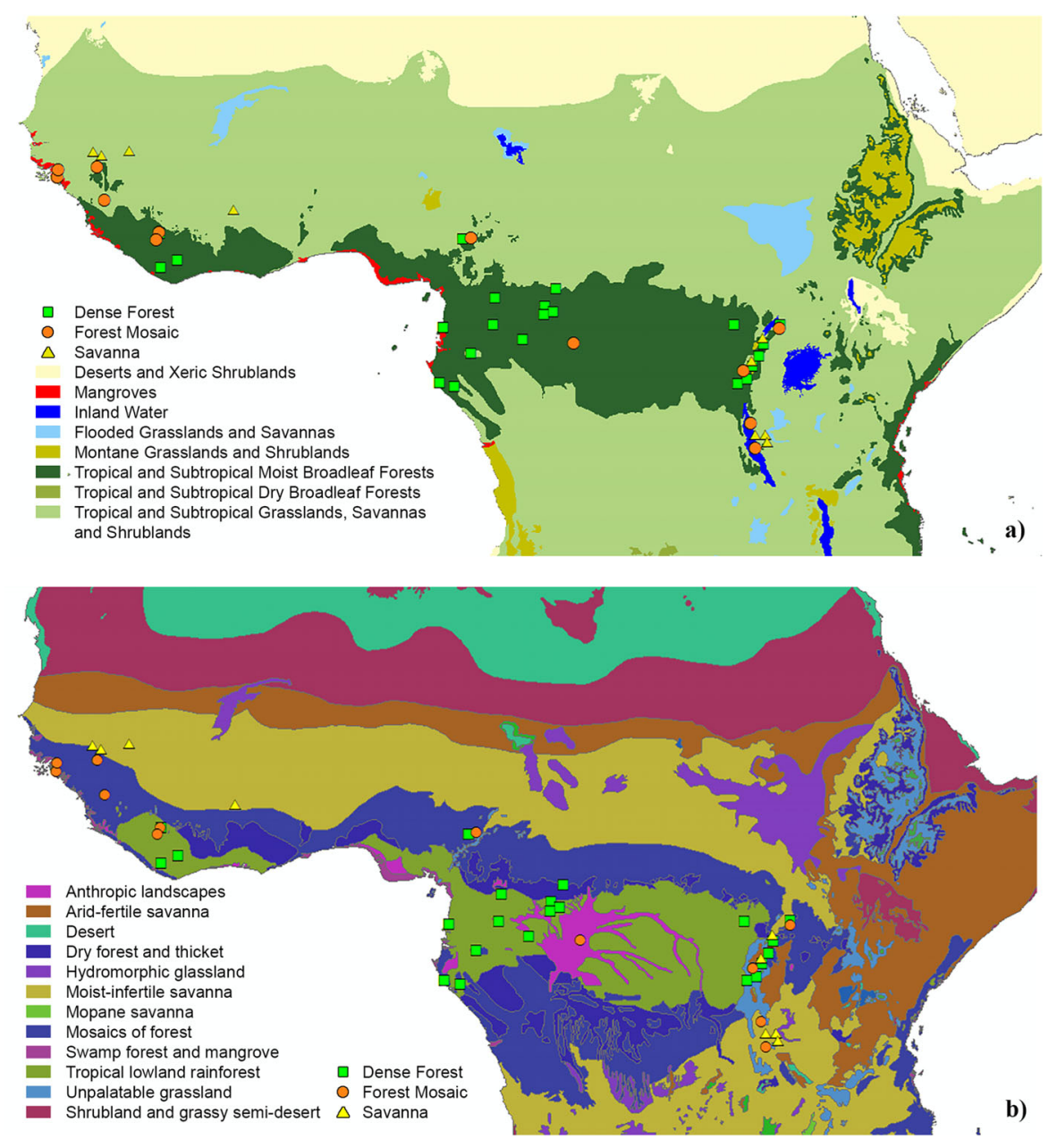

Fig. 2 Chimpanzee study sites overlaid on (a) the WWF terrestrial ecoregions (WWF 2018), (b) White's Vegetation Map of Africa (White 1983), and (c) the Whittaker Biome Diagram (Ricklefs 2008; Whittaker 1975). Sites are labeled as dense forest, forest mosaic, and savanna based on terminology or descriptions of vegetation cover by researchers. 


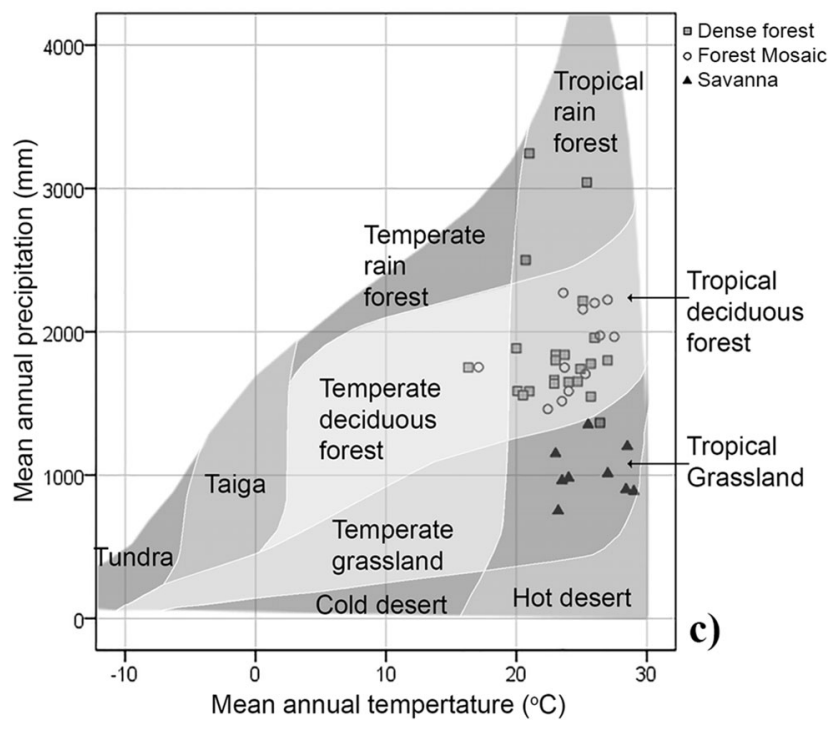

Fig. 2 (continued).

environmental data associated with these researcher categories, although the inclusion of data from additional chimpanzee study sites could clarify how distinctive these classes are. We could not formally quantify environmental boundaries for the chimpanzee habitat categories of dense forest, forest mosaic, and savanna, due to overlapping ranges of the environmental variables assessed.

We found that chimpanzee researcher classifications did not match consistently with the bioclimatic categorizations of the WWF terrestrial ecoregions (WWF 2018), White's Vegetation Map of Africa (White 1983), and the Whittaker Biome Diagram (Ricklefs 2008; Whittaker 1975). In particular, the plot of chimpanzee study sites, labeled with their researcher classifications, against the bioclimatic classification of Whittaker showed some outliers (Fig. 2c). The dense forest and forest mosaic outliers in the "Temperate deciduous forest" biome (i.e., Bwindi-Impenetrable National Park in Uganda, and Tongo in DRC) are likely a consequence of high altitudes and associated lower mean annual temperatures at these sites (Kajobe and Roubik 2006; Lanjouw 2002; Stanford and O'Malley 2008), whereas the dense forest outlier within the "Tropical grassland" biome (i.e., Dzanga-Ndoki National Park in CAR) likely reflects the relatively low mean annual rainfall at this forested site (Blom et al. 2001). Sites identified by chimpanzee researchers as savannas generally matched with grassland or savanna classifications of the assessed biome, vegetation and climate classification schemes, but dense forest and forest mosaic sites inconsistently fell into several, noncorresponding classes (including grassland and savanna categories) within the WWF terrestrial ecoregions, White's Vegetation Map of Africa, and the Whittaker Biome Diagram. Differences are likely due to the scale of measurement and details of the environmental classifications in these often global classification schemes. Whereas existing biome maps focus on quantifying the broad-scale environments of the world, researchers studying chimpanzees focus on environmental classifications from a chimpanzee perspective at a more local scale. These illustrative examples thus show that 
Table III Chimpanzee study sites, labeled with the landscape classification used by researchers, in relation to the landscape classifications of the WWF terrestrial ecoregions (WWF 2018), White's Vegetation Map of Africa (White 1983), and the Whittaker Biome Diagram (Ricklefs 2008; Whittaker 1975).

\begin{tabular}{|c|c|c|c|c|c|}
\hline \multirow[t]{2}{*}{$\begin{array}{l}\text { Bioclimatic } \\
\text { classification }\end{array}$} & \multirow[t]{2}{*}{ Habitat class } & \multicolumn{4}{|c|}{$\begin{array}{l}\text { Chimpanzee researcher-specified } \\
\text { landscape class }\end{array}$} \\
\hline & & Savanna & $\begin{array}{l}\text { Forest } \\
\text { mosaic }\end{array}$ & $\begin{array}{l}\text { Dense } \\
\text { forest }\end{array}$ & Total \\
\hline \multirow{4}{*}{$\begin{array}{l}\text { WWF terrestrial } \\
\text { ecoregions }\end{array}$} & Tropical and subtropical moist broadleaf forest & 0 & 5 & 19 & 24 \\
\hline & $\begin{array}{l}\text { Tropical and subtropical grasslands, savannas, } \\
\text { and shrublands }\end{array}$ & 9 & 5 & 3 & 17 \\
\hline & Mangroves & 0 & 2 & 0 & 2 \\
\hline & Total & 9 & 12 & 22 & 43 \\
\hline \multirow{9}{*}{$\begin{array}{l}\text { White's Vegetation } \\
\text { Map of Africa }\end{array}$} & Tropical lowland rainforest & 0 & 2 & 11 & 13 \\
\hline & Dry forest and thicket & 0 & 0 & 1 & 1 \\
\hline & Swamp forest and mangrove & 0 & 2 & 0 & 2 \\
\hline & Mosaics of forest & 0 & 4 & 2 & 6 \\
\hline & Arid-fertile savanna & 1 & 0 & 2 & 3 \\
\hline & Moist-infertile savanna & 8 & 1 & 0 & 9 \\
\hline & Unpalatable grassland & 0 & 2 & 5 & 7 \\
\hline & Anthropic landscapes & 0 & 1 & 1 & 2 \\
\hline & Total & 9 & 12 & 22 & 43 \\
\hline \multirow{5}{*}{$\begin{array}{l}\text { Whittaker Biome } \\
\text { Diagram }\end{array}$} & Tropical rainforest & 0 & 0 & 3 & 3 \\
\hline & Tropical deciduous forest & 0 & 11 & 17 & 28 \\
\hline & Temperate deciduous forest & 0 & 1 & 1 & 2 \\
\hline & Tropical grassland & 9 & 0 & 1 & 10 \\
\hline & Total & 9 & 12 & 22 & 43 \\
\hline
\end{tabular}

landscape classifications of chimpanzee study sites used by researchers differ from the ecological definitions set out by the three selected biome classification schemes.

Rather than identifying quantifiable natural groupings and nonoverlapping chimpanzee habitat categories, our analyses showed that the environmental data from the 43 chimpanzee study sites followed an environmental gradient. The chimpanzee study sites were spread across the range of each environmental variable assessed. Based on observed gradients of mean annual temperature, mean annual rainfall, rainfall seasonality, forest cover, and Hansen tree cover in the PCA, researcher-classified savanna sites consistently fell at one end of the environmental continuum, dense forest sites fell typically at the other end of the continuum, and forest mosaic sites fell in the middle. Outliers and overlap in this environmental continuum can likely be explained by anthropogenic influences: The dense forest outlier in the bottom left panel of Fig. 3 (i.e., Gishwati in Rwanda) likely fell into the savanna-mosaic side of the environmental continuum as this site represents a forest island amidst a human-dominated landscape and therefore has relatively low forest cover and Hansen tree cover (Chancellor et al. 2012a; Chancellor et al. 2017). Similarly, the two forest mosaic sites that fell closest to the chimpanzee researcher-classified savanna sites in Fig. 3 (i.e., Gombe in Tanzania 


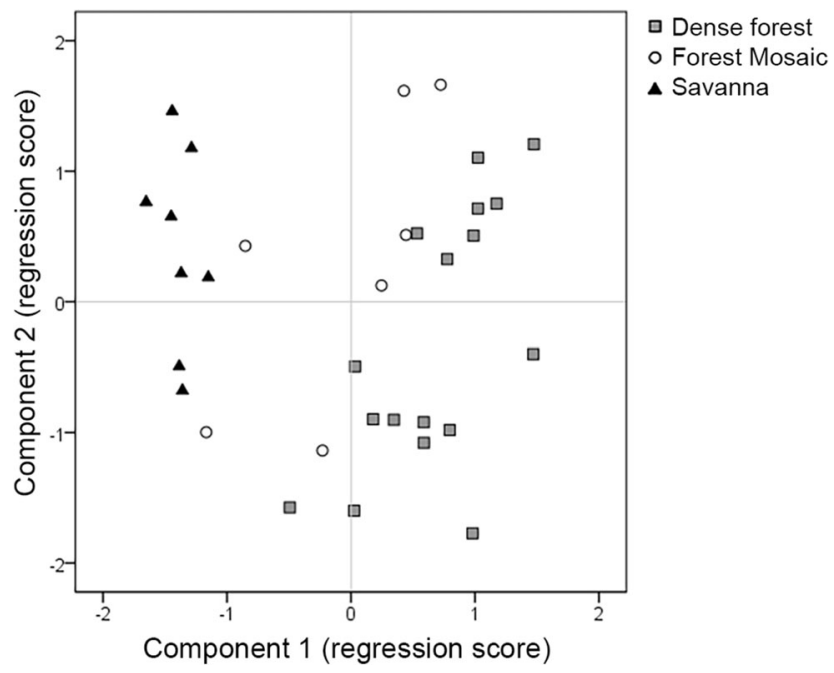

Fig. 3 Regression component scores (component 1 and component 2) of chimpanzee study sites used in a principal components analysis (PCA). Sites are labeled as dense forest, forest mosaic, and savanna based on terminology or descriptions of vegetation cover by researchers. These labels are indicative only and were not used as input for the PCA.

and Bulindi in Uganda) are characterized by relatively low forest cover as a result of anthropogenic disturbance (e.g., McLennan and Ganzhorn 2017; Pusey et al. 2007). The currently used forest-savanna dichotomy thus masks a progression of environmental adaptation for chimpanzees, and we argue that the inclusion of an additional, intermediate "forest mosaic" category is more meaningful than focusing only on the ends of this environmental gradient, while also reflecting a better appreciation of the gradient itself. By acknowledging intermediate habitats and recognizing a "forest mosaic" category for chimpanzees, we propose that researchers in future studies define the position of their study site to the middle or end of the forest-savanna gradient, rather than to one end only.

We found that sites with higher mean annual temperatures and longer dry seasons were more likely to be classified as forest mosaics by chimpanzee researchers than

Table IV Results of a principal components analysis (PCA) of the habitat at chimpanzee study sites

\begin{tabular}{lll}
\hline Environmental variable & Component $1^{\mathrm{a}}$ & Component $2^{\mathrm{a}}$ \\
\hline Forest cover $(\%)$ & 0.941 & \\
Mean annual precipitation $(\mathrm{mm})$ & 0.791 & -0.416 \\
Hansen tree cover $(\%)$ & 0.743 & 0.891 \\
Longest consecutive dry season (\# months) & -0.302 & 0.846 \\
Total number of dry months $(\#)$ & & 0.665 \\
Mean annual temperature $\left({ }^{\circ} \mathrm{C}\right)$ & & 1.064 \\
Eigenvalue & 3.343 & 17.731 \\
Variance explained $(\%)$ & 55.725 & \\
\hline
\end{tabular}

a Small loading coefficients between -0.3 and 0.3 suppressed. 
dense forests, even if they had high forest cover (Figs. 1 and 3). There are two possible explanations for this observation. First, this could indicate that forests in areas with longer dry seasons and higher temperatures are different from forests in areas with shorter dry seasons and lower temperatures. These differences could indicate a change from (semi-)deciduous to evergreen forest types (Saha 2012). Indeed, some studies of chimpanzee forest mosaic sites included a reference to the semideciduous character of at least part of the forest in their field site descriptions (Caiquene-Cadique: Sa et al. 2013; Gashaka Gumti: Fowler and Sommer 2007; Gombe: Bakuza and Nkwengulila 2009, Gilby et al. 2006; Lac Tumba Landscape: Inogwabini et al. 2012; Mahale: Kaburu and Newton-Fisher 2015; Matsusaka et al. 2006; Nakamura et al. 2013). Semideciduous forests typically shed their leaves at certain times of year and their microhabitat characteristics differ between "leaf-off" and "leaf-on" conditions (as derived from, e.g., Hue et al. 2016; Rakotomalala et al. 2017). For example, microhabitat characteristics such as temperatures and luminosities typically increase, and canopy cover, amount of shade, and the presence of food sources typically decrease, when trees shed their leaves. Therefore, (semi-)deciduous forests may potentially be periodically less favorable for primates [as shown, for example, for red-handed howlers (Alouatta belzebul) and marmosets (Callithrix jacchus): Hue et al. 2016; red-tailed sportive lemurs (Lepilemur ruficaudatus): Rakotomalala et al. 2017; and spider monkeys (Ateles geoffroyi): Chapman et al. 1995]. Thus, a relationship between forest cover, annual temperature, and length of the longest consecutive dry season may influence the apparent mosaic (and potentially deciduous) character of chimpanzee study sites. Dense forest and forest mosaic sites may sometimes have similar percentages of forest cover, but the accompanying temperature and rainfall seasonality may differentiate these forests as habitat. Primatologists should describe the deciduous nature of their field sites to identify the role deciduousness plays in primate habitat suitability and survival.

Second, the finding that chimpanzee dense forest and forest mosaic sites sometimes overlap in forest cover percentage may indicate that different researchers use different approaches to classify their landscapes. For example, researchers at Mahale Mountains, Caiquene-Cadique, and Gashaka Gumti classify their sites as forest mosaics despite relatively high forest cover (Bessa et al. 2015; Nakamura et al. 2015; Sommer et al. 2012, 2016). These sites fell relatively close to the researcher-classified dense forest sites in our scatterplots (Fig. 1) and principal components analysis (Fig. 3, top right panel). Scientists currently use many different terminologies to assess global-scale landscapes and different vegetation types at a more local scale (Domínguez-Rodrigo 2014; Gardner 2006; McGrew et al. 1981; Moore 1992; Torello-Raventos et al. 2013; White 1983), and various interpretations of what constitutes a "forest" or "savanna" vegetation type or landscape exist (Domínguez-Rodrigo 2014; Gardner 2006; McGrew et al. 1981; Oliveras and Malhi 2016; Torello-Raventos et al. 2013; White 1983). This again emphasizes the need for consistent environmental definitions and terminologies for primates across the globe, and we argue that future primatological studies should provide detailed descriptions of vegetation and (micro-)climate characteristics at their field sites for transparency, clarity, and facilitation of future comparative efforts and classification attempts.

Although our analyses did not show natural groupings across environmental variables for researcher-derived chimpanzee habitat categories, additional data are needed 
for future analyses. For example, whereas we only focused on basic environmental metrics in our review of chimpanzee habitat classifications, other factors, such as anthropogenic influences and additional environmental parameters, might help in further distinguishing between chimpanzee landscapes. Although the importance of basic environmental variables in chimpanzee habitat distinctions has been acknowledged (Abwe et al. 2019; Loudon et al. 2016; McGrew et al. 1981; Moore 1992), chimpanzee abundance in anthropogenic habitats can be strongly influenced by factors such as hunting pressure and the presence of and distance to roads (Boesch et al. 2017; Heinicke et al. 2019), while chimpanzee abundance in savannas can be affected by variables such as habitat heterogeneity, canopy cover, and floral species richness (Wessling et al. unpubl. data). The Whittaker Biome Diagram (Ricklefs 2008; Whittaker 1975) furthermore separated out two sites (i.e., Bwindi-Impenetrable National Park in Uganda: Kajobe and Roubik 2006; Stanford and O'Malley 2008; and Tongo in DRC: Lanjouw 2002) at high elevations, which may be an important additional factor to consider. These factors may thus provide additional variables for chimpanzee landscape classifications and may increase the total variance explained by the principal components analysis.

Future classification attempts would furthermore benefit from greater precision in site location data. We used GPS-referenced locations of chimpanzee study sites and 5$\mathrm{km}$ buffers for our analyses of Hansen tree cover (Hansen et al. 2013). Although the GPS-referenced locations and 5-km buffers were based on published chimpanzee literature [ESM Appendices S1-S3], their exact values are often not in the center of the chimpanzee home range. Frequently, the specified GPS coordinates represent the location of the research camp or National Park/Forest Reserve (e.g., Chancellor et al. 2012b; Russak 2013), which are rarely situated in the heart of the chimpanzee territory, and other researchers provide only the corners of their study area or National Park (e.g., Ogawa et al. 2014; Stanford and O’Malley 2008). As a result, our Hansen tree cover values may not have reflected the precise chimpanzee home range at each site. This is sometimes also observed for researcher-derived vegetation cover when researchers specify the cover of the National Park/Forest Reserve instead of the actual chimpanzee home range (ESM Appendix S1). Additionally, although selected for uniformity, our 5$\mathrm{km}$ buffer may not be equally appropriate for each site, because chimpanzee home range sizes vary across study sites, and the Hansen tree cover percentages may include areas outside the actual chimpanzee home range, such as water bodies, agricultural fields, and settlements. Slight deviations in site location and home range size relative to the actual chimpanzee home range at study sites might explain the discrepancies observed between Hansen tree cover and forest cover defined by researchers. Satellite-derived measures of tree cover provide objective measurements of vegetation for comparative analyses, and if researchers want to use the various available vegetation and climate layers based on satellite data, we urge the collection and publication of detailed information on the actual centroid location, spread, and size of the chimpanzee home range area at study sites.

Our review of vegetation and climate at chimpanzee dense forest, forest mosaic, and savanna sites focused on 43 field study sites for which our systematic literature search in Web of Science provided sufficient data for analysis (i.e., information on basic vegetation data to allow for landscape class distinctions, as well as climate data or location). These 43 sites do not represent all chimpanzee study sites, or the entire 
biogeographical chimpanzee range, and site selection thus influenced the values included in our analyses. At least 120 additional chimpanzee study sites (Heinicke et al. 2019; Kühl et al. 2019; Tagg et al. 2017) were not included in our analyses due to insufficient data. Future inclusion of additional chimpanzee study sites requires the publication of data on annual temperature, annual rainfall, rainfall seasonality, researcher-derived forest cover, and Hansen tree cover to establish further understanding of the environmental gradient in which chimpanzees occur and to test whether the proposed environmental continuum for chimpanzee landscapes from savanna to forest mosaic to dense forest encompasses the full variety of environmental conditions in which chimpanzees can range.

Published literature on chimpanzees thus emphasizes a forest-savanna chimpanzee distinction (as reviewed in, e.g., Hunt and McGrew 2002; McGrew et al. 1981; Moore 1996), and we argue for the inclusion of an additional, intermediate "forest mosaic" category and the acknowledgment of the environmental gradient that chimpanzees have adapted to occupy. However, rather than applying these labels to the chimpanzees themselves, we furthermore propose that these labels be applied directly to the chimpanzee habitat. Instead of discussing "forest chimpanzees", "savanna chimpanzees", and now perhaps "mosaic chimpanzees", we argue that researchers should discuss "chimpanzees in dense forest/forest mosaic/ savanna habitat". Chimpanzees inhabit forest to savanna environments throughout their range, and this variety of habitats is observed for all four chimpanzee subspecies (e.g., Humle et al. 2016). While the terms "forest chimpanzees", "savanna chimpanzees", and "mosaic chimpanzees" might imply to some that these are different species, as is, for example, the case with African forest elephants (Loxodonta cyclotis) and African savanna elephants (Loxodonta africana: Ishida et al. 2011; Roca et al. 2001), the description of "chimpanzees in dense forest/forest mosaic/ savanna habitat" may provide a more realistic and careful alternative. We recommend that future studies provide detailed descriptions of the vegetation cover and climate at their chimpanzee study sites, and position their sites along the savanna-forest continuum for transparency, clarity, and consistency in research and comparative assessments.

\section{Conclusion}

Despite the wide use of the forest-savanna chimpanzee distinction in published literature, clear definitions of these landscapes for chimpanzees based on environmental variables at study sites or determined in relation to existing bioclimatic classifications are lacking. Based on explicitly used terminology or descriptions of vegetation cover, we showed that chimpanzee researchers classified their sites as either forest or savanna. However, we recognized a further distinction within forest sites between dense forests and forest mosaics, which is not acknowledged within the current forest-savanna dichotomy. We observed no natural groupings in environmental data for 43 chimpanzee study sites and it proved impossible to formally quantify environmental boundaries for the researcher-based classifications of dense forest, forest mosaic, and savanna sites into nonoverlapping habitat categories. This was due to overlap among categories in the environmental variables assessed and inconsistencies with the bioclimatic categorizations of Whittaker, the WWF terrestrial ecoregions, and White's Vegetation Map of 
Africa. Rather, we found that chimpanzee study sites fell along an environmental continuum from savannas to dense forests, with forest mosaics in between. The dichotomy of forest and savanna chimpanzees therefore masks the environmental gradient that chimpanzees have adapted to occupy, and much of the environmental gradient is currently contained within a generic and undefined forest chimpanzee category. We argue that recognizing an additional, intermediate category of forest mosaic habitat is a more meaningful reflection of the environmental adaptations for chimpanzees than focusing only on the ends of this environmental gradient. Although categorization of habitat is typically a simplification of the natural world, science benefits from clear and detailed categories in order to provide structure and consistency between different researchers. For clarity and consistency, we recommend that future studies acknowledge this environmental continuum for chimpanzees, identify where on the environmental gradient their study sites fall, and provide detailed environmental data on vegetation cover and climate at their study sites to support this.

Acknowledgments This research was supported by a PhD studentship from Bournemouth University (BU) and the Institute for Studies on Landscape and Human Evolution (ISLHE) to K. L. van Leeuwen, and forms part of the research program LEAP: Landscape Ecology and Primatology. We thank K. Koops (University of Cambridge), V. Reynolds (University of Oxford), and A. Pascual-Garrido (University of Oxford) for their responses to the questionnaire on the environmental determinants of chimpanzee site selection for specific activities during the $\mathrm{PhD}$ study of $\mathrm{K}$. L. van Leeuwen, which were used here for gathering environmental data for specific chimpanzee study sites. We thank J. Moore for reviewing an earlier version of this manuscript and providing useful comments. We thank E. G. Wessling, J. M. Setchell, and the anonymous reviewers for helpful comments and constructive feedback on the manuscript.

Author Contributions KL, RH, and AK conceived and designed the study, analyzed the data, and interpreted the results. KL wrote the manuscript. RH and AK reviewed the manuscript, data analyses, and interpretation and provided editorial advice.

Open Access This article is licensed under a Creative Commons Attribution 4.0 International License, which permits use, sharing, adaptation, distribution and reproduction in any medium or format, as long as you give appropriate credit to the original author(s) and the source, provide a link to the Creative Commons licence, and indicate if changes were made. The images or other third party material in this article are included in the article's Creative Commons licence, unless indicated otherwise in a credit line to the material. If material is not included in the article's Creative Commons licence and your intended use is not permitted by statutory regulation or exceeds the permitted use, you will need to obtain permission directly from the copyright holder. To view a copy of this licence, visit http://creativecommons.org/licenses/by/4.0/.

\section{References}

Abwe, E. E., Morgan, B. J., Tchiengue, B., Kentatchime, F., Doudja, R., et al (2019). Habitat differentiation among three Nigeria-Cameroon chimpanzee (Pan troglodytes ellioti) populations. Ecology and Evolution, 9, 1489-1500.

Arroyo-Rodríguez, V., \& Fahrig, L. (2014). Why is a landscape perspective important in studies of primates? American Journal of Primatology, 76, 901-909.

Bakuza, J. S., \& Nkwengulila, G. (2009). Variation over time in parasite prevalence among free-ranging chimpanzees at Gombe National Park, Tanzania. International Journal of Primatology, 30, 43-53.

Bastin, D. (1996). Foret de N'gotto inventaire forestier: Rapport general, zone 1 et 2. Rapport ECOFAC, RCA. Paris: Union Européen, Groupement AGRECO/ CIRAD Foret. 
Bessa, J., Sousa, C., \& Hockings, K. J. (2015). Feeding ecology of chimpanzees (Pan troglodytes verus) inhabiting a forest-mangrove-savanna-agricultural matrix at Caiquene-Cadique, Cantanhez National Park, Guinea-Bissau. American Journal of Primatology, 77, 651-665.

Blasco, F., Whitmore, T. C., \& Gers, C. (2000). A framework for the worldwide comparison of tropical woody vegetation types. Biological Conservation, 95, 175-189.

Blom, A., Almaši, A., Heitkönig, I. M. A., Kpanou, J. B., \& Prins, H. H. T. (2001). A survey of the apes in the Dzanga-Ndoki National Park, Central African Republic: A comparison between the census and survey methods of estimating the gorilla. African Journal of Ecology, 39, 98-105.

Boesch, L., Mundry, R., Kühl, H., \& Berger, R. (2017). Wild mammals as economic goods and implications for their conservation. Ecology and Society, 22, 36.

Bryson-Morrison, N., Matsuzawa, T., \& Humle, T. (2016). Chimpanzees in an anthropogenic landscape: Examining food resources across habitat type at Bossou, Guinea, West Africa. American Journal of Primatology, 78, 1237-1249.

Chancellor, R. L., Langergraber, K., Ramirez, S., Rundus, A. S., \& Vigilant, L. (2012a). Genetic sampling of unhabituated chimpanzees (Pan troglodytes schweinfurthii) in Gishwati Forest Reserve, an isolated forest fragment in Western Rwanda. International Journal of Primatology, 33, 479-488.

Chancellor, R. L., Rundus, A. S., \& Nyandwi, S. (2012b). The influence of seasonal variation on chimpanzee (Pan troglodytes schweinfurthii) fallback food consumption, nest group size, and habitat use in Gishwati, a Montane rain forest fragment in Rwanda. International Journal of Primatology, 33, 115-133.

Chancellor, R. L., Rundus, \& Nyandwi, S. (2017). Chimpanzee seed dispersal in a montane forest fragment in Rwanda. American Journal of Primatology, 79, e22624.

Chapman, C. A., \& Peres, C. A. (2001). Primate conservation in the new millennium: The role of scientists. Evolutionary Anthropology, 10, 16-33.

Chapman, C. A., Wrangham, R. W., \& Chapman, L. J. (1995). Ecological constraints on group size: An analysis of spider monkey and chimpanzee. Behavioral Ecology and Sociobiology, 36, 59-70.

Collins, D. A., \& McGrew, W. C. (1988). Habitats of three groups of chimpanzees (Pan troglodytes) in western Tanzania compared. Journal of Human Evolution, 17, 553-574.

Copeland, S. R. (2009). Potential hominin plant foods in northern Tanzania: Semi-arid savannas versus savanna chimpanzee sites. Journal of Human Evolution, 57, 365-378.

Domínguez-Rodrigo, M. (2014). Is the "savanna hypothesis" a dead concept for explaining the emergence of the earliest hominins? Current Anthropology, 55, 59-81.

Estrada, A., Garber, P., Rylands, A. B., Roos, C., Fernandez-Duque, E., et al (2017). Impeding extinction crisis of the world's primates: Why primates matter. Science Advances, 3, 1-16.

Estrada, A., Raboy, B. E., \& Oliveira, L. C. (2012). Agroecosystems and primate conservation in the tropics: A review. American Journal of Primatology, 74, 696-711.

Fick, S. E., \& Hijmans, R. J. (2017). WorldClim 2: New $1 \mathrm{~km}$ spatial resolution climate surfaces for global land areas. International Journal of Climatology, 37, 4302-4315.

Fowler, A., \& Sommer, V. (2007). Subsistence technology of Nigerian chimpanzees. International Journal of Primatology, 28, 997-1023.

Galán-Acedo, C., Arroyo-Rodríguez, V., Andresen, E., Arregoitia, L. V., Vega, E., et al (2019). The conservation value of human-modified landscapes for the world's primates. Nature Communications, $10,152$.

Gardner, T. A. (2006). Tree-grass coexistence in the Brazilian cerrado: Demographic consequences of environmental instability. Journal of Biogeography, 33, 448-463.

Gilby, I. C., Eberly, L. E., Pintea, L., \& Pusey, A. E. (2006). Ecological and social influences on the hunting behaviour of wild chimpanzees, Pan troglodytes schweinfurthii. Animal Behaviour, 72, 169-180.

Hansen, M. C., Potapov, P. V., Moore, R., Hancher, M., Turubanova, S. A., et al (2013). High-resolution global maps of 21st-century forest cover change. Science, 342, 850-853.

Heinicke, S., Mundry, R., Boesch, C., Amarasekaran, B., Barrie, A., et al (2019). Characteristics of positive deviants in Western chimpanzee populations. Frontiers in Ecology and Evolution, 7, 16.

Hernandez-Aguilar, R. A. (2009). Chimpanzee nest distribution and site reuse in a dry habitat: Implications for early hominin ranging. Journal of Human Evolution, 57, 350-364.

Hernandez-Aguilar, R. A., Moore, J., \& Pickering, T. R. (2007). Savanna chimpanzees use tools to harvest the underground storage organs of plants. PNAS, 104, 19210-19213.

Hijmans, R. J., Cameron, S. E., Parra, J. L., Jonges, P. G., \& Jarvis, A. (2005). Very high resolution interpolated climate surfaces for global land areas. International Journal of Climatology, 25, 1965-1978.

Hijmans, R. J. \& Elith, J. (2017). Species distribution modeling with R. R CRAN Project.

Howard, P. C. (1991). Nature conservation in Uganda's tropical forest reserves. Gland, Switzerland and Cambridge, UK: IUCN and WWF-International. 
Hue, T., Caubet, M., \& Moura, A. C. A. (2016). Howlers and marmosets in Pacatuba: An overcrowded existence in a semi-deciduous Atlantic forest fragment? Mammalia, 81, 339-348.

Humle, T., Maisels, F., Oates, J. F., Plumptre, A., \& Williamson, E. A. (2016). Pan troglodytes. (Errata version published in 2016). The IUCN Red List of Threatened Species 2016: e.T15933A102326672.

Hunt, K. D., \& McGrew, W. C. (2002). Chimpanzees in the dry habitats of Assirik, Senegal and Semliki Wildlife Reserve, Uganda. In C. Boesch, G. Hohmann, \& L. Marchant (Eds.), Behavioural diversity in chimpanzees and bonobos (pp. 35-51). Cambridge: Cambridge University Press.

Inogwabini, B. I., Abokome, M., Kamenge, T., Mbende, L., \& Mboka, L. (2012). Preliminary bonobo and chimpanzee nesting by habitat type in the northern Lac Tumba landscape, Democratic Republic of Congo. African Journal of Ecology, 50, 285-298.

Inskipp, T. (2005). Chimpanzees (Pan troglodytes). In J. Caldecott \& L. Miles (Eds.), World atlas of great apes and their conservation (pp. 53-81). London: University of California Press.

Ishida, Y., Demeke, Y., van Coeverden de Groot, P. J., Georgiadis, N. J., Leggett, K. E. A., et al (2011). Distinguishing forest and savanna African elephants using short nuclear DNA sequences. Journal of Heredity, 102, 610-616.

Kaburu, S. S. K., \& Newton-Fisher, N. E. (2015). Trading or coercion? Variation in male mating strategies. Behavioural Ecology and Sociobiology, 69, 1039-1052.

Kajobe, R., \& Roubik, D. W. (2006). Honey-making bee colony abundance and predation by apes and aumans in a Uganda Forest Reserve. Biotropica, 38, 210-218.

Koops, K., McGrew, W. C., de Vries, J., \& Matsuzawa, T. (2012). Nest-building by chimpanzees (Pan troglodytes verus) at Seringbara, Nimba Mountains: Antipredation, thermoregulation, and antivector hypotheses. International Journal of Primatology, 33, 356-380.

Kortlandt, A. (1983). Marginal habitats of chimpanzees. Journal of Human Evolution, 12, 231-278.

Kühl, H. S., Boesch, C., Kulik, L., Haas, F., Arandjelovic, M., et al (2019). Human impact erodes chimpanzee behavioral diversity. Science, 363, 1453-1455.

Lanjouw, A. (2002). Behavioural adaptations to water scarcity in Tongo chimpanzees. In C. Boesch, G. Hohmann, \& L. Marchant (Eds.), Behavioural diversity in chimpanzees and bonobos (pp. 52-60). Cambridge: Cambridge University Press.

Loudon, J. E., Sandberg, P. A., Wrangham, R. W., Fahey, B., \& Sponheimer, M. (2016). The stable isotope ecology of Pan in Uganda and beyond. American Journal of Primatology, 78, 1070-1085.

Matsuzawa, T., Humle, T., \& Sugiyama, Y. (2011). The chimpanzees of Bossou and Nimba. Tokyo: Springer.

Matsusaka, T., Nishie, H., Shimada, M., Kutsukake, N., Zamma, K., et al (2006). Tool-use for drinking water by immature chimpanzees of Mahale: Prevalence of an unessential behavior. Primates, 47, 113-122.

McGarigal, K. (2002). Landscape pattern metrics. In A. H. El-Shaarawi \& W. W. Piegorsch (Eds.), Encyclopedia of environmetrics (pp. 1135-1142). Chichester: Wiley.

McGarigal, K., Tagil, S., \& Cushman, S. A. (2009). Surface metrics: An alternative approach to patch metrics for the quantification of landscape structure. Landscape Ecology, 24, 433-450.

McGrew, W. C., Baldwin, P. J., \& Tutin, C. E. G. (1981). Chimpanzees in a hot, dry and open habitat: Mt. Asserik, Senegal, West Africa. Journal of Human Evolution, 10, 227-244.

McGrew, W. C., Baldwin, P. J., \& Tutin, C. E. G. (1988). Diet of wild chimpanzees (Pan troglodytes verus) at Mt. Assirik, Senegal: I. Composition. American Journal of Primatology, 16, 213-226.

McKinney, T. (2015). A classification system for describing anthropogenic influence on nonhuman primate populations. American Journal of Primatology, 77, 715-726.

McLennan, M. R., \& Ganzhorn, J. U. (2017). Nutritional characteristics of wild and cultivated foods for chimpanzees (Pan troglodytes) in agricultural landscapes. International Journal of Primatology, 38, 122150 .

Meijaard, E. (2016). The role of multifunctional landscapes in primate evolution. In S. A. Wich \& A. J. Marshall (Eds.), An introduction to primate conservation (pp. 205-218). Oxford: Oxford University Press.

Moore, J. (1992). 'Savanna’ chimpanzees. In T. Nishida, W. C. McGrew, P. Marler, M. Pickford, \& F. B. M. de Waal (Eds.), Topics in primatology, Vol. I: Human origins (pp. 99-118). Tokyo: University of Tokyo Press.

Moore, J. (1996). Savanna chimpanzees, referential models and the last common ancestor. In W. C. McGrew, L. Marchant, \& T. Nishida (Eds.), Great ape societies (pp. 275-292). Cambridge: Cambridge University Press.

Nakamura, M., Corp, N., Fujimoto, M., Fujita, S., Hanamura, S., et al (2013). Ranging behavior of Mahale chimpanzees: A 16 year study. Primates, 54, 171-182.

Nakamura, M., Hosaka, K., Itoh, N., \& Zamma, K. (2015). Mahale chimpanzees: 50 years of research. Cambridge: Cambridge University Press. 
Ogawa, H., Yoshikawa, M., \& Idani, G. (2014). Sleeping site selection by savanna chimpanzees in Ugalla, Tanzania. Primates, 55, 269-282.

Oliveras, I., \& Malhi, Y. (2016). Many shades of green: The dynamic tropical forest-savannah transition zones. Philosophical Transactions of the Royal Society B: Biological Sciences, 371, 1-15.

Peel, M. C., Finlayson, B. L., \& McMahon, T. A. (2007). Updated world map of the K"oppen-Geiger climate classification. Hydrology and Earth System Sciences, 11, 1633-1644.

Piel, A. K., Strampelli, P., Greathead, E., Hernandez-Aguilar, R. A., Moore, J., \& Stewart, F. A. (2017). The diet of open-habitat chimpanzees (Pan troglodytes schweinfurthii) in the Issa valley, western Tanzania. Journal of Human Evolution, 112, 57-69.

Pruetz, J. D. (2007). Evidence of cave use by savanna chimpanzees (Pan troglodytes verus) at Fongoli, Senegal: Implications for thermoregulatory behavior. Primates, 48, 316-319.

Pruetz, J. D. (2018). Nocturnal behavior by a diurnal ape, the West African chimpanzee (Pan troglodytes verus), in a savanna environment at Fongoli, Senegal. American Journal of Physical Anthropology, 166, $541-548$.

Pruetz, J. D., \& Bertolani, P. (2007). Savanna chimpanzees, Pan troglodytes verus, hunt with tools. Current Biology, 17, 412-417.

Pruetz, J., \& Bertolani, P. (2009). Chimpanzee (Pan troglodytes verus) behavioral responses to stresses associated with living in a savannah-mosaic environment: Implications for hominin adaptations to open habitats. PaleoAnthropology, 252-262.

Pruetz, J. D., Fulton, S. J., Marchant, L. F., McGrew, W. C., Schiel, M., \& Waller, M. (2008). Arboreal nesting as anti-predator adaptation by savanna chimpanzees (Pan troglodytes verus) in southeastern Senegal. American Journal of Primatology, 70, 393-401.

Pruetz, J. D., Marchant, L. F., Arno, J., \& McGrew, W. C. (2002). Survey of savanna chimpanzees (Pan troglodytes verus) in southeastern Senegal. American Journal of Primatology, 58, 35-43.

Pusey, A. E., Pintea, L., Wilson, M. I., Kamenya, S., \& Goodall, J. (2007). The contribution of long-term research at Gombe National Park to chimpanzee conservation. Conservation Biology, 21, 623-634.

Rakotomalala, E. J., Rakotondraparany, F., Perofsky, A. C., \& Lewis, R. J. (2017). Characterization of the tree holes used by Lepilemur ruficaudatus in the dry, deciduous forest of Kirindy Mitea National Park. Folia Primatologica, 88, 28-41.

Ricklefs, R. (2008). Ecology: The economy of nature (6th ed.). New York: W. H. Freeman and Company.

Roca, A. L., Georgiadis, N., Pecon-Slattery, J., \& O’Brien, S. J. (2001). Genetic evidence for two species of elephant in Africa. Science, 293, 1473-1477.

Russak, S. M. (2013). Ecological role of dry-habitat chimpanzees (Pan troglodytes schweinfurthii). $\mathrm{PhD}$ thesis, Arizona State University.

Sa, R. M., Petrasova, J., Pomajbikova, K., Profousova, I., Petrzelkova, K. J., et al (2013). Gastrointestinal symbionts of chimpanzees in Cantanhez National Park, Guinea-Bissau with respect to habitat fragmentation. American Journal of Primatology, 75, 1032-1041.

Saha, P. (2012). Modern climatology. New Delhi, India: Allied Publishers.

Sommer, V., Buba, U., Jesus, G., \& Pascual-Garrido, A. (2012). Till the last drop: Honey gathering in Nigerian chimpanzees. Ecotropica, 18, 55-64.

Sommer, V., Buba, U., Jesus, G., \& Pascual-Garrido, A. (2016). Sustained myrmecophagy in Nigerian chimpanzees: Preferred or fallback food? American Journal of Physical Anthropology, 162, 328-336.

Stanford, C. B., \& O'Malley, R. C. (2008). Sleeping tree choice by Bwindi chimpanzees. American Journal of Primatology, 70, 642-649.

Tagg, N., McCarthy, M., Dieguez, P., Bocksberger, G., Willie, J., (2017). Nocturnal activity in wild chimpanzees (Pan troglodytes): Evidence for flexible sleeping patterns and insights into human evolution. American Journal of Physical Anthropology, 166, 510-529.

Thomas, S. G. (2016). The dictionary of physical geography (4th ed.). Chichester: John Wiley \& Sons.

Torello-Raventos, M., Feldpausch, T. R., Veenendaal, E., Schrodt, F., Saiz, G., et al (2013). On the delineation of tropical vegetation types with an emphasis on forest/savanna transitions. Plant Ecology \& Diversity, 6 , 101-137.

Wessling, E. G., Deschner, T., Mundry, R., Pruetz, J. D., Wittig, R. M., \& Kühl, H. S. (2018a). Seasonal variation in physiology challenges the notion of chimpanzees (Pan troglodytes verus) as a forest-adapted species. Frontiers in Ecology and Evolution, 6, 60.

Wessling, E. G., Kühl, H. S., Mundry, R., Deschner, T., \& Pruetz, J. D. (2018b). The costs of living at the edge: Seasonal stress in wild savanna-dwelling chimpanzees. Journal of Human Evolution, 121, 1-11.

Wessling, E. G., Oelze, V. M., Eshuis, H., Pruetz, J. D., \& Kühl, H. S. (2019). Stable isotope variation in savanna chimpanzees (Pan troglodytes verus) indicate avoidance of energetic challenges through dietary compensation at the limits of the range. American Journal of Physical Anthropology, 168, 665-675. 
White, F. (1983). The vegetation of Africa: A descriptive memoir to accompany the Unesco/AETFAT/ UNSO vegetation map of Africa. Natural resources research $X X$. Paris: Courvoisier S.A.

Whittaker, R. H. (1975). Communities and ecosystems (2nd ed.). New York: Macmillan.

WWF (World Wildlife Fund) (2018). Terrestrial ecoregions. https://www.worldwildlife.org/biomecategories/terrestrial-ecoregions. GIS map of TNC's terrestrial ecoregions of the world available online at http://maps.tnc.org/gis_data.html, published by The Nature Conservancy.

\section{Affiliations}

\section{Kelly L. van Leeuwen ${ }^{1,2,3} \cdot$ Ross A. Hill ${ }^{1,2}$ - Amanda H. Korstjens ${ }^{1,2,3}$}

1 Department of Life and Environmental Sciences, Bournemouth University, Poole, UK

2 Landscape Ecology and Primatology (LEAP), Bournemouth University, Poole, UK

3 Institute for Studies on Landscape and Human Evolution (ISLHE), Bournemouth University, Poole, UK 